\title{
A note-question on partitions of semigroups
}

\author{
Igor Protasov, Ksenia Protasova
}

\begin{abstract}
Given a semigroup $S$ and an $n$-partition $\mathcal{P}$ of $S, n \in \mathbb{N}$, do there exist $A \in \mathcal{P}$ and a subset $F$ of $S$ such that $S=F^{-1}\{x \in S: x A \bigcap A \neq \emptyset\}$ and $|F| \leq n$ ?

We give an affirmative answer provided that either $S$ is finite or $n=2$.
\end{abstract}

2010 MSC: 20M10, 05D10.

Keywords: Partitions of semigroups, covering number.

\section{Introduction}

In 1995, the first author asked the following question [3, Problem 13.44].

Given a group $G$ and an $n$-partition $\mathcal{P}, n \in \mathbb{N}$ of $G$, do there exist $A \in \mathcal{P}$ and a subset $F$ of $G$ such that $G=F A A^{-1}$ and $|F| \leq n$ ?

For the current state of this open problem see the survey [1]. We mention only that an answer is positive if either $G$ is amenable (in particular, finite), or $n \leq 3$, or $x^{-1} A x=A$ for any $A \in \mathcal{P}$ and $x \in G$. If $G$ is an arbitrary group and $\mathcal{P}$ is an $n$-partition of $G$ then one can choose $A, B \in \mathcal{P}$ and subsets $F, H$ of $G$ such that $G=F A A^{-1},|F| \leq n$ ! and $G=H B B^{-1} B$ and $|H| \leq n$.

In this note, we formulate a semigroup version of above question and give positive answer provided that either $S$ is finite or $n=2$.

For systematic exposition of Ramsey theory of semigroups see [2].

For a semigroup $S, a \in S, A \subseteq S$ and $B \subseteq S$, we use the standard notations

$$
a^{-1} B=\{x \in S: a x \in B\}, \quad A^{-1} B=\bigcup_{a \in A} a^{-1} B .
$$

We set $\Delta(A)=\{x \in S: x A \bigcap A \neq \emptyset\}$ and observe that $\Delta(A)=\left\{x \in S: x^{-1} A \bigcap A \neq \emptyset\right\}$ and if $S$ is a group then $\Delta(A)=A A^{-1}$.

We suppose that $S^{-1} A=S$ and define a covering number

$$
\operatorname{cov} A=\min \left\{|X|: X \subseteq S, S=X^{-1} A\right\} .
$$

If $S^{-1} A \neq S$ then $\operatorname{cov} A$ is not defined. Clearly, $\operatorname{cov} A$ is defined if and only if $S x \bigcap A \neq \emptyset$ for every $x \in S$.

Now we are ready for promised question. $n$ ?

Given a semigroup $S$ and an n-partition $\mathcal{P}$ of $S$, does there exist $A \in \mathcal{P}$ such that $\operatorname{cov} \Delta(A) \leq$

\section{Results}

Theorem 1. For a semigroup $S$ and an n-partition $\mathcal{P}$ of $S$, there exists $A \in \mathcal{P}$ such that $\operatorname{cov} \Delta(A) \leq 2^{2^{n-1}-1}$. 
If $n=2$ then $\operatorname{cov} \Delta(A) \leq 2$. In a personal communication, $\mathrm{G}$. Bergman answered the question positively for $n=3$, and noticed that, we may suppose that a semigroup $S$ is a monoid.

Theorem 2. For a finite semigroup $S$ and an n-partition $\mathcal{P}$ of $S$, there exists $A \in \mathcal{P}$ such that $\operatorname{cov} \Delta(A) \leq n$.

Theorem 3. If a subset $A$ of a semigroup $S$ contains either left or right zero then $\operatorname{cov} \Delta(A)=$ 1.

\section{Proofs}

Proof of Theorem 1. We adopt arguments from [4, pp. 120-121] proving this theorem for groups.

We define a function $f: \mathbb{N} \times \mathbb{N} \longrightarrow \mathbb{N}$ by

$$
f(1, m)=m \text { and } f(n+1, m)=f\left(n, m+m^{2}\right) .
$$

By [4, Lemma 12.2], $f(n, m) \leq 2^{2^{n-1}-1} m^{2^{n-1}}$.

We use induction on $n$ to prove the following auxiliary statement

(*) Let $F, A_{1}, A_{2}, \ldots, A_{n}$ be subsets of a semigroup $S$ such that $S=F^{-1}\left(A_{1} \cup A_{2} \cup \cdots \cup A_{n}\right)$ and $|F| \leq m$. Then there exist $i \in\{1,2, \ldots, n\}$ and a subset $K$ of $S$ such that $S=K^{-1} \Delta\left(A_{i}\right)$ and $|K| \leq f(n, m)$.

For $n=1$, we have $S=F^{-1} A_{1}$. We take an arbitrary $x \in S$ and choose $g \in F$ such that $x A_{1} \bigcap g^{-1} A_{1} \neq \emptyset$. Then $A_{1} \bigcap x^{-1} g^{-1} A_{1} \neq \emptyset, A_{1} \bigcap(g x)^{-1} A_{1} \neq \emptyset$ so $g x \in \Delta(A), x \in g^{-1} \Delta(A)$, $x \in F^{-1} \Delta(A)$ and $S=F^{-1} \Delta(A)$.

Let $S=F^{-1}\left(A_{1} \bigcup A_{2} \bigcup \ldots \cup A_{n+1}\right)$. We consider two cases.

Case 1. $g A_{1} \subseteq F^{-1}\left(A_{2} \cup \ldots \cup A_{n+1}\right)$ for some $g \in S$. Then $A_{1} \subseteq g^{-1} F^{-1}\left(A_{2} \ldots A_{n+1}\right)$ and $S=\left(F^{-1} \bigcup F^{-1} g^{-1} F^{-1}\right)\left(A_{2} \bigcup \cdots \cup A_{n+1}\right)$. Since $|F \cup F g F| \leq m+m^{2}$, by the inductive hypothesis, there exist $i \in\{2,3, \ldots, n+1\}$ and a subset $K$ of $S$ such that

$$
S=K^{-1} \Delta\left(A_{i}\right), \quad|K| \leq f\left(n, m+m^{2}\right)=f(n+1, m) .
$$

Case 2. $x A_{1} \cap F^{-1} A_{1} \neq \emptyset$ for every $x \in S$. Then $A_{1} \cap x^{-1} F^{-1} A_{1} \neq \emptyset, x^{-1} g^{-1} A_{1} \bigcap A_{1} \neq \emptyset$ for some $g \in F, g x \in \Delta\left(A_{1}\right)$ and $x \in g^{-1} \Delta(A), S=F^{-1} \Delta(A)$. We set $K=F$ and note that $|K| \leq m \leq f(n+1, m)$.

To conclude the proof, we assume that $S=A_{1} \cup \ldots \cup A_{n}$, take an arbitrary $g \in S$, put $F=\{g\}$, note that $S=F^{-1}\left(A_{1} \cup \ldots \cup A_{n}\right)$ and apply $(*)$.

Proof of Theorem 2. Let $S$ be a finite semigroup and $S=A_{1} \cup \ldots \cup A_{n}$. We take a minimal right ideal $R$ of $S$, choose $r \in S$ and note that $r S \subseteq R, S \subseteq r^{-1} R$, so we may suppose that $S=R$. By [2, Theorem 1.63(g)], $S$ is a direct product of a group $G$ and a right zero semigroup $I$. We take $a \in I$ and put $H=G \times\{a\}$. For each $i \in\{1, \ldots, n\}$, we denote $B_{i}=A_{i} \bigcap H$. Since $H$ is a finite group, there are $j \in\{1, \ldots, n\}$ and $K \in H$ such that $|K| \leq n$ and $H=K^{-1} \Delta_{H}\left(B_{j}\right)$, where $\Delta_{H}\left(B_{j}\right)=\left\{x \in H: x B_{j} \cap B_{j} \neq \emptyset\right\}$. We take an arbitrary $(g, b) \in G \times I$ and choose 
$z \in K$ such that $z(g, a) \in\left\{x \in H: x B_{j} \cap B_{j} \neq \emptyset\right\}$. Since $I$ is a right zero semigroup, we have $z(g, b) B_{j} \bigcap B_{j} \neq \emptyset$. Hence $(g, b) \in z^{-1}\left\{x \in S: x A_{j} \bigcap A_{j} \neq \emptyset\right\}$ and $S=K^{-1} \Delta\left(A_{j}\right)$.

Proof of Theorem 3. If $a \in A$ is left zero then, for every $x \in S$, we have $S=a^{-1} a=a^{-1}\{x \in$ $A: x A \bigcap A \neq \emptyset\}$ and $S=a^{-1} \Delta(A)$.

If $a \in A$ is right zero then, for every $x \in S, a \in x A \bigcap A$ so $x A \bigcap A \neq \emptyset$ and $S=\Delta(A)$ and $S=g^{-1} \Delta(A)$ for each $g \in S$.

Acknowledgement. We thank George Bergman for constructive remarks on the seminal version of this note.

\section{References}

[1] Banakh, T., Protasov, I., Slobodianiuk, S. Densities, submeasures and partitions of Gspaces and groups, Algebra and Discrete Mathematics, 17 (2014), Number 2, 193-221, preprint (http://arxiv.org./abs/1303.4612).

[2] N. Hindman, D. Strauss, Algebra in the Stone-ČCch Compactification, 2nd edition, de Gruyter, 2012.

[3] V.D. Mazurov, E.I. Khukhro (eds), Unsolved problems in group theory, the Kourovka notebook, 13-th augmented edition, Novosibirsk, 1995.

[4] Protasov I., Banakh T., Ball structures and colorings of groups and graphs, Math. Stud. Monogr. Ser., Vol.11, VNTL, Lviv, 2003.

Department of Cybernetics, Kyiv University,

Prospect Glushkova 2, corp. 6,

03680 Kyiv, Ukraine

e-mail: I.V.Protasov@gmail.com

Ksenia Protasova

Department of Cybernetics, Kyiv University,

Prospect Glushkova 2, corp. 6,

03680 Kyiv, Ukraine

e-mail: ksuha@freenet.com.ua 